\title{
Estudio comparativo de factores de resiliencia en docentes de contextos socialmente vulnerables
}

\section{Comparative study related to resilience factors generated on teachers who work in vulnerable contexts}

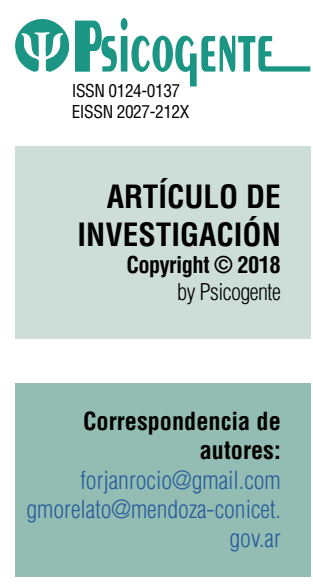

Recibido: 03-04-17 Aceptado: $17-08-17$ Publicado: $01-07-18$

\author{
Rocío Forján iD \\ Universidad del Aconcagua, Mendoza, Argentina \\ Gabriela Morelatto iD \\ Instituto de Ciencias Humanas, Sociales y Ambientales (INCIHUSA)
}

Resumen

Objetivo: Analizar y comparar factores de resiliencia en docentes de nivel primario de la provincia de Mendoza, Argentina, quienes se desempeñaban en contextos con características de vulnerabilidad social y sin ella. A partir de los aportes teóricos de Henderson y Milstein, se consideraron las variables: participación significativa, conducta prosocial, autoestima-aprendizaje y percepción de apoyo.

Método: Se realizó un estudio mixto, descriptivo-comparativo, con diseño no experimental, y transversal. La muestra contó con 66 docentes, de los cuales 34 trabajaban en contextos socialmente vulnerables, y 32 en contextos sin vulnerabilidad. Los instrumentos utilizados fueron: la Escala de Resiliencia Docente y una Entrevista ad hoc.

Resultados: Indicaron diferencias significativas en el factor conducta prosocial, presentando puntajes más altos los docentes de contextos vulnerables, y una tendencia a la significación en los factores percepción de apoyo y participación significativa, a favor del mismo grupo. Esto señala la importancia del apoyo mutuo, el trabajo en equipo y el respaldo institucional como factores protectores.

Conclusiones: Las características adversas del contexto pueden generar riesgos y dificultades en el quehacer; simultáneamente, funcionan como potenciadoras de procesos de resiliencia.

Palabras clave: resiliencia, vulnerabilidad social, docencia, factores protectores, factores de riesgo.

Abstract

Objective: This paper aims to analyze and compare factors of resilience in primary level teachers who perform in context with and without social vulnerability features, from Provincia de Mendoza, Argentina. This study is based on theoretical contributions of Henderson and Milstein, the variables considered were as follows: meaningful involvement, pro-social behavior, self-steem, learning and support perception.

Method: This study was conducted based on a quantitative, descriptive and comparative method, and non-experimental and transverse design. 66 teachers were sampled, as follow: 34 of them were working in socially vulnerable contexts and 32 teachers were working on contexts without vulnerability. Teacher Resilience Scale and a Semi-structured Interview were used as instruments.

Result: Significant differences in pro-social behavior factors were evident, where teachers in vulnerable contexts show higher scores and a trend towards significance on perceived support and meaningful participation factor, in favor of their own group. These results show the importance of mutual support, teamwork and institutional support as protective factors.

Conclusions: The adverse context features can generate risks and difficulties on teachers' daily work simultaneously; these features function as enhancing resilience processes.

Key words: resilience; social vulnerability; teaching, protective factors, risk factors 


\section{INTRODUCCIÓN}

Este trabajo se sustenta en estudios previos que examinaron factores de resiliencia en niños y familias en circunstancias de riesgo (Morelato, 2011a; 2011b, 2014; Pinto Cortez, 2014). También se basa en trabajos preliminares enfocados en la evaluación de aspectos cognitivos, emocionales y socio-familiares en niños y familias de contextos socialmente vulnerables (Greco, Morelato e Ison, 2007; Ison, Morelato, Carrada, Espósito, Greco, Maddio y Korzeniowski, 2007; Korzeniowski, 2011; Canto, Morelato y Greco, 2013; Escarbajal-Frutos, Izquierdo-Rus y López-Martínez, 2014).

La vulnerabilidad social es una condición que se asocia a factores que generan indefensión y debilitamiento de las potencialidades y capacidades en algunos grupos sociales. De este modo, siguiendo a Golovanevsky (2007a; 2007b), se la entiende como el resultado de una relación dinámica entre los recursos que facilitan el desempeño del individuo en la sociedad y los requerimientos de las estructuras de acceso al bienestar brindado por el mercado, el Estado y la comunidad. Si bien se sabe que las condiciones de vulnerabilidad social impactan negativamente el desarrollo infantil en casi todas las áreas (Ison, 2004; Colombo y Lipina, 2005; Colombo, 2007; McClelland, Cameron, Connor, McDonald, Carrie, Jewkes \& Morrison, 2007; Segretin, Lipina y Colombo, 2007; Lacunza, Castro-Solano y Contini, 2009; Rosemberg y Stein, 2009; Valdés Arriagada y Spencer Contreras, 2011; Marzonetto, 2015), en años recientes se ha desarrollado una nueva forma de estudiar las problemáticas de riesgo, basada en el abordaje de los recursos individuales y del entorno. Esta perspectiva no se opone al modelo de riesgo, sino que lo complementa y lo enriquece (Munist, Santos y Kotliarenco, 1998; Herscovici Raush, 2011; Ríos y Andes, 2016). Además, esta línea pertenece al enfoque salugénico, el cual propone estudiar los comportamientos humanos focalizando los recursos y no los déficits (Keyes, Shmotkin y Ryff, 2002 citados en Greco, Morelato e Ison, 2007; Mariñelarena-Dondena, 2016)-, Justamente el estudio de la resiliencia pertenece a esta perspectiva.

Los trabajos recientes sobre la resiliencia ya no proponen considerarla como un factor individual ni de personalidad, sino como un engranaje relacional y ecosistémico, que permite encontrar oportunidades para evitar el estancamiento o deterioro (Gómez y Kotliarenco, 2010; Bravo y Lopez, 2015). En este engranaje, se incluyen múltiples factores individuales y contextuales, los cuales pueden ser tanto protectores como de riesgo. De esta forma, la resiliencia es vista como un proceso dinámico, que depende de factores internos (personales) y externos (contextuales), tanto de riesgo como protec- 
tores. Ambos factores interactúan entre sí para generar un mecanismo que posibilita la continuación del desarrollo de algunos aspectos a pesar de las circunstancias, lo que se entiende como una adaptación positiva (Morelato, 2011a; 2014)

Por otra parte, el contexto escolar, aun a pesar de las dificultades relacionadas con aspectos de presupuesto y políticas educativas en América Latina, sigue siendo un organismo protector. Esto se debe a que la escuela no solamente cumple funciones educativas sino también de socialización y formación de valores humanos, entre otras cosas. Sin embargo, actualmente muchas escuelas estatales que reciben población de entornos vulnerables atraviesan una gran diversidad de problemáticas sociales que se han ido agudizando. Por lo tanto, sus educadores deben sortear una gran cantidad de dificultades, que los lleva a poner en marcha una serie de recursos personales y contextuales frente a diversas situaciones de riesgo cotidianas. De este modo, como plantea Cyrulnik (2009), el docente puede convertirse en un guía o tutor de resiliencia. Sin embargo, esto solo puede darse si él mismo ha desarrollado a su vez mecanismos de resiliencia, ya que ello influye de modo importante en el fortalecimiento de las habilidades de los estudiantes (Aguaded Gómez y Almeida Pires Cavaco, 2016; Henderson y Milstein, 2003; Pérez, García, Gil y Caballer, 2010).

En este sentido, estudios previos indican que la resiliencia en docentes es un proceso individual, relacional y colectivo. Y más en concreto, una capacidad latente, dinámica y sujeta a fluctuación, donde los factores internos y externos se vinculan con la capacidad de proponerse desafíos, tener vocación, bienes personales y relaciones con los estudiantes, los padres, los colegas y la escuela. Estos autores también señalan que los maestros que se esfuerzan por enseñar al máximo en estas circunstancias, requieren de resiliencia emocional, la cual implica ser capaz de manejar los desafíos para lograr el éxito, es decir, que el individuo progrese más allá del uso de estrategias de afrontamiento.

En Perú, Guerra (2010) indagó especialmente los factores de resiliencia en docentes de su país y concluyó que existen diferencias significativas en los niveles de resiliencia entre los docentes de nivel inicial, primaria y secundaria, alcanzando los primeros mayores puntajes y los de primaria, menor resiliencia. Tal hallazgo arroja un interrogante sobre lo que ocurre en este sector de docentes. 
Por otra parte, en Argentina existen muy pocos trabajos en esta temática para corroborar y comparar, aunque sea de modo preliminar, cuáles son los aspectos que ejercen mayor influencia en el desarrollo de la resiliencia en los maestros. Esto permitiría tener un panorama más actualizado de lo que ocurre en este país, a fin de analizar y contrastar con lo que sucede en toda Latinoamérica y en otros países respecto a la resiliencia de docentes en el ámbito educativo.

Por todo lo planteado, nuestro objetivo general fue analizar y comparar factores asociados a la resiliencia en una muestra de docentes de nivel primario de la provincia de Mendoza de dos entornos sociales diferentes. Los objetivos específicos fueron: describir factores de resiliencia en docentes que desempeñan su labor en contextos socialmente vulnerables; explorar los mismos factores en docentes que trabajan en contextos sin estas características y, finalmente, comparar las diferencias entre los grupos previamente mencionados.

\section{MÉTODO}

\subsection{Diseño}

Se trató de un estudio mixto con prevalencia del enfoque cuantitativo, con alcance descriptivo y de tipo comparativo, para lo cual se utilizó un diseño de tipo no experimental, transversal (Hernández Sampieri, Fernández Collado, Baptista Lucio, 2010).

\subsection{Participantes}

La muestra seleccionada fue de tipo no probabilística y de carácter intencional (Hernández Sampieri, et al., 2010). Debido a esto, las conclusiones de este trabajo solo pueden ser generalizadas a poblaciones que cuenten con características similares a las de los casos investigados.

Se trabajó con 66 docentes, de los cuales 34 trabajan en dos establecimientos educativos de la provincia, pertenecientes a contextos socialmente vulnerables, según el criterio de la Dirección General de Escuelas (DGE, 2017), y con los que se integró un primer grupo. Las mismas reciben población de barrios periféricos caracterizados por factores como precariedad laboral y dificultades en al acceso a recursos económicos, sociales, de salud y educación. El otro grupo, constituido por 32 docentes, desempeña su labor en contextos sin esta característica y recibe población de un nivel socioeco- 
nómico medio y medio-bajo, pero con ninguna o menores dificultades en el acceso al trabajo, la salud, educación, redes sociales y oportunidades de desarrollo. La muestra contó con 8 participantes de sexo masculino (12 \%) y 58 ( $88 \%)$ de sexo femenino, con edades que oscilaban entre los 28 y 58 años ( $M=41.79 D=7.282$ ) y un predominio del rango de edad entre los 40 y 49 años (47\%). De la totalidad de los participantes ( $N=66)$ el $51.5 \%$ trabajan en contextos socialmente vulnerables y $48.5 \%$ en contextos sin vulnerabilidad. A su vez, trabajan entre 11 y 20 horas semanales (36.4\%) y presentan una antigüedad laboral de entre 11 y 20 años (Tabla 1). La distribución según sexo y contexto puede observarse en la Tabla 2.

\subsection{Instrumentos}

Se utilizaron dos instrumentos: la Escala de Resiliencia Docente (E.R.D.) y una entrevista semiestructurada.

\subsubsection{Escala de resiliencia docente (E.R.D) (Guerra, 2013)}

Este instrumento cuenta con 69 reactivos, con cinco alternativas de respuesta posibles, donde 5 corresponde a "totalmente de acuerdo" y 1 a "totalmente en desacuerdo". El mismo fue seleccionado por ser un instrumento relevante y congruente con la concepción de resiliencia adoptada. Según los estudios realizados por su autor, los resultados alcanzados en el análisis factorial exploratorio a través del método de los componentes principales y el método de rotación Oblimin, establecen que la medida de adecuación del muestreo de Kaiser-Meyer-Olkin alcanzó un valor de .962, que se considera adecuado. Por otro lado, el test de esfericidad de Bartlett presenta un valor que es significativo ( $B=22169.096 ; p<0.000$ ). En este sentido, los resultados del análisis indican que cuatro factores permiten explicar el $40.43 \%$ de la varianza total. El primer factor explicó el 19.68 \% de la varianza total de los ítems que corresponden al área de participación significativa. El segundo factor explicó el $11.78 \%$ en el área de conducta prosocial. El tercero, el $4.61 \%$ de autoestima y aprendizaje; mientras que el cuarto explicó el $4.35 \%$ del área de percepción y apoyo. Debido a que esta escala no cuenta con estudios de baremación en Argentina, para nuestra investigación en un $\mathrm{N}$ pequeño, solo se pudo realizar en la correspondiente adaptación léxica y de contenido. Para esto se utilizó el criterio de jueces expertos, obteniendo un índice de concordancia de 0.83 p $<0.001$, lo cual también se considera adecuado (Aron y Aron, 2001). Respecto de la confiabilidad de la escala para nuestro estudio se obtuvo un Alpha de Cronbach de 0.85 para la escala total, mientras que los resultados 
de los factores fueron: Factor 1: 0.849; Factor 2: 0.699; Factor 3: 0.142; Factor 4: 0.765 . Siguiendo estos datos, podemos considerar que la escala total es confiable, al igual que los resultados de los factores 1, 2 y 4 . En cuanto al factor 3 , el puntaje nos indica una baja confiabilidad. Esto sugiere que los ítems que conforman el factor en nuestra población deberían revisarse en posteriores estudios. Por otra parte, la escala se basa en los conceptos teóricos de Henderson y Milstein (2003). De esta manera, los ítems se distribuyen en 4 áreas consideradas como factores de resiliencia docente, que se describen a continuación:

- Participación significativa (38 ítems): Implica conceder responsabilidad en la toma de decisiones y la resolución de problemas (Gamboa, 2008 citado por López Hidalgo, 2012). Se relaciona con situaciones como participar en actividades cooperativas, intervenir en la elaboración y la modificación de las políticas y las reglas, sentirse valorado en el desempeño de su rol, valorar el aumento de responsabilidad del establecimiento educativo como medio de asegurar la intervención del docente en la adopción de decisiones, dedicar su tiempo y adquirir las habilidades requeridas para participar con eficacia, estar al tanto de lo que sucede y tomar parte en los éxitos de la escuela. En presencia de este factor, el docente crea espacios significativos que facilitan el encuentro y en los que no hay límites; entiende que necesita espacios de los que apropiarse para desarrollar su protagonismo y sentido de pertenencia (Guerra, 2010).

- Conducta prosocial (18 ítems): Es aquella conducta social positiva con o sin motivación altruista. Los docentes resilientes responden más al contacto con otros seres humanos y generan más respuestas positivas en las otras personas; además, son activos, flexibles y adaptables. Este componente incluye cualidades como la de estar listo para responder a cualquier estímulo, comunicarse con facilidad, demostrar empatía y afecto, y tener comportamientos prosociales. Concretamente, esta área mide si el docente busca oportunidades de interactuar con otros, si presta ayuda y la recibe de otros educadores, si tiene sensación de pertenencia a través de mensajes de valoración y apoyo, o si demuestra confianza en su propio potencial de excelencia y en el de los demás (Guerra, 2010).

- Autoestima y aprendizaje (5 ítems): En virtud de este factor, el docente manifiesta tener una elevada autoestima, promovida por oportunidades de aprendizaje. La autoestima y el aprendizaje de competencias personales, especialmente las habilidades vinculadas con la interacción con los otros, son factores constructores de resiliencia, que en la práctica 
educativa se pueden potenciar con estrategias o actuaciones como poseer una buena opinión del propio desempeño profesional, dedicar el tiempo suficiente al aprendizaje, entre otros (Guerra, 2010).

- Percepción de apoyo (8 ítems): Esta área mide si el docente percibe que la comunidad respalda sus actividades, y si se siente apoyado por las autoridades en el cumplimiento de sus expectativas laborales. El objetivo último de este reconocimiento y valoración ha de ser que los docentes se concienticen sobre sus potencialidades (Guerra, 2010).

\subsubsection{Entrevista semidirigida}

Se realizó una entrevista ad hoc (Siquier de Ocampo et al., 1997) con el objetivo de explorar aspectos relacionados con la percepción del docente en cuanto a la diferencia en cada tipo de contexto. Este instrumento se implementó a fin de complementar los resultados obtenidos por el cuestionario y establecer un buen rapport previo. Las preguntas de la entrevista fueron las siguientes: 1- ¿Trabaja doble turno?; 2- ¿Cuántos años lleva como docente? ¿Cuántos años lleva trabajando en esta escuela?; 3- ¿Ha trabajado en algún establecimiento que se encuentre en otro contexto social?; 4- En caso positivo, ¿qué encuentra como diferente o significativo entre dichos establecimientos? Por su naturaleza, las respuestas 1,2 y 3, simplemente se cuantificaron. La pregunta 4 se categorizó tal como se describe en el apartado de análisis de los resultados.

\subsection{Procedimiento}

Para llevar a cabo el estudio, en primer lugar, se solicitó autorización de ingreso a las autoridades de las escuelas presentándoles, para ello, el proyecto de investigación y la metodología de trabajo propuesta. Luego de aprobado el trabajo, se procedió a contactar a los docentes y se obtuvo el consentimiento informado por escrito de cada uno de ellos. Luego se administró la entrevista semidirigida y la escala de resiliencia. En última instancia, se llevó a cabo el análisis y discusión de los datos obtenidos.

\subsection{Análisis de los datos}

El análisis estadístico de los resultados se efectuó utilizando procedimientos paramétricos y, en concreto, el programa estadístico Statistical Package for Social Sciences (SPSS), versión 22.0. Tales procedimientos se ejecutaron debido a que se obtuvieron valores de asimetría y curtosis adecuados en los 4 factores, que permitieron su utilización. Los puntajes resultantes oscilaron entre -1.029 y 1.895 (Aron y Aron, 2001). 
Se realizó un análisis descriptivo univariado en términos de distribución de frecuencias para responder a los objetivos 1 y 2 del estudio. Además, se calcularon 3 rangos en cada factor de la escala para considerar el nivel de resiliencia de los docentes en cada uno de ellos (bajo, medio y alto). El criterio para la conformación de rangos fue la utilización de la medida de posición conocida como cuartiles. En este análisis, se utilizó la prueba chi cuadrado. Por otra parte, se incorporó un análisis bivariado mediante la prueba t de diferencias entre medias para muestras independientes, a fin de responder al tercer objetivo del estudio. Se fijó un nivel de significación equivalente a 0.05.

En el caso de las entrevistas, se realizó un análisis de contenido y luego se cuantificaron los datos en las categorías surgidas. Para el análisis de contenido se tuvieron en cuenta las fases de análisis, constituidas en primer lugar por el descubrimiento y, en segundo lugar, por la codificación, categorización y reestructuración de categorías. El proceso realizado fue el siguiente: a- Se realizó la fase de descubrimiento a través de la lectura y relectura del corpus de los datos extraídos de las entrevistas. Posteriormente, estas se recategorizaron y ajustaron a una codificación cuantitativa; b-Se utilizó el índice de K (Kappa) de Cohen para evaluar el acuerdo entre jueces con respecto a la confiabilidad de las categorías emergentes. Para tal fin se utilizó una grilla de respuesta tipo Likert con valores del 1 al 3, donde el valor 1 es considerado inadecuado, 2 medianamente adecuado y 3 adecuado. Se obtuvo un índice de concordancia de $0.42 p=0.027$, lo cual es considerado moderadamente aceptable puesto que se juzgan como aceptables valores de $\mathrm{k}$ mayores $\mathrm{o}$ iguales a 0.40 (Landis \& Koch, 1977, citado en Aron y Aron, 2001).

\subsection{Conflicto de intereses}

La presente investigación se realizó con la participación de docentes de diferentes establecimientos educativos de la Provincia de Mendoza, quienes, luego de la evaluación, accedieron a los resultados obtenidos para planificar acciones futuras dentro de su institución, tales como estrategias grupales y factores a reforzar, entre otras.

En uno de los casos, la escuela consideró los datos emanados del trabajo para evidenciar la importancia del apoyo profesional en la institución, obteniendo como resultado el aporte económico estatal necesario para solventar las intervenciones 


\section{RESULTADOS}

Tabla 1.

Variables sociodemográficas de los docentes evaluados. Distribución por frecuencia y porcentaje

\begin{tabular}{cccc}
\hline & DATOS DESCRIPTIVOS DE LA MUESTRA & F & $\%$ \\
\hline \multirow{2}{*}{ Contextos } & Contextos socialmente vulnerables & 34 & 51.5 \\
& Contextos no vulnerables & 32 & 48.5 \\
Sexo & Femenino & 58 & 88 \\
Trabaja doble turno & Masculino & 8 & 12 \\
& Contextos socialmente vulnerables & 25 & 60 \\
Horas semanales que & Contextos no vulnerables & 17 & 40 \\
trabaja & 01-10 horas & 2 & 3 \\
& $11-20$ horas & 24 & 36.4 \\
& $21-30$ horas & 17 & 25.8 \\
Años como docente & $31-40$ horas & 19 & 28.8 \\
& $41-50$ horas & 4 & 6 \\
& $01-10$ años & 25 & 37.9 \\
\hline
\end{tabular}

Nota: $n=66$

En la Tabla 1, se puede observar la distribución por frecuencia y porcentaje de las variables sociodemográficas de los docentes evaluados, que componen el total de la muestra.

Tabla 2.

Distribución en términos de frecuencia y porcentaje en los docentes evaluados según sexo y tipo de contexto.

\begin{tabular}{cccc}
\hline \multirow{2}{*}{ SEXo } & \multicolumn{3}{c}{ CONTEXTO } \\
\cline { 2 - 4 } & Socialmente Vulnerable & No Vulnerable & Total \\
\hline Masculino & $11.8 \%(4)$ & $12.5 \%(5)$ & $12.1 \%(8)$ \\
Femenino & $88.2 \%(30)$ & $87.5 \%(28)$ & $87.9 \%(58)$ \\
Total & $100.0 \%(34)$ & $100.0 \%(32)$ & $100.0 \%(9)$ \\
\hline
\end{tabular}

La Tabla 2 registra la distribución en términos de frecuencia y porcentaje de los docentes evaluados según sexo y tipo de contexto. Se puede observar que el mayor porcentaje en ambos contextos se concentra en el sexo femenino.

A continuación, se describen los factores de resiliencia en términos de porcentaje en la escala aplicada a docentes de ambos contextos. 


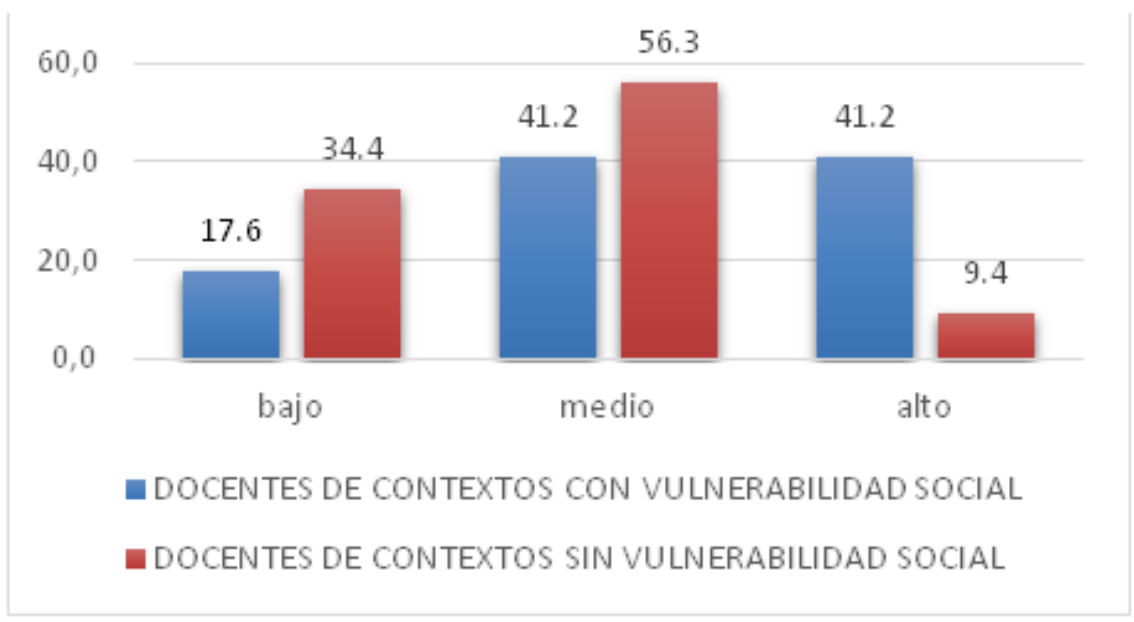

Gráfico 1. Resultados de factor 1, "Participación significativa” según contexto

El Gráfico 1 evidencia que los docentes de contextos con vulnerabilidad presentan valores elevados en los rangos alto y medio ( $41.2 \%$ en cada rango). En cambio, los docentes de contextos sin vulnerabilidad social presentan un nivel predominantemente medio en el factor (56.3\%), seguido por el nivel bajo (34.3\%). La prueba de chi cuadrado señaló que estas diferencias son significativas $\left(\chi^{2}=9.03 p<0.0011\right.$ Tau $\left.c=-0.360\right)$

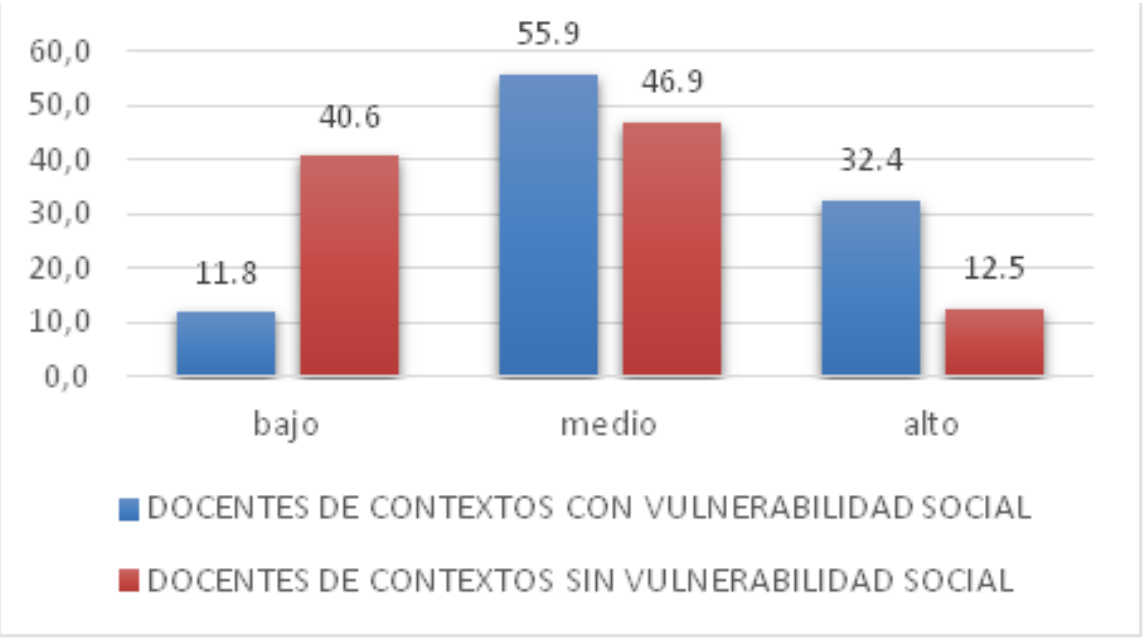

Gráfico 2. Resultados factor 2, "Conducta Prosocial", según el contexto

En el Gráfico 2 se observa que los docentes de contextos vulnerables presentan principalmente un nivel medio en el factor de conducta prosocial (55.9\%), 
seguido por el nivel alto (32.4\%). Por su parte, los docentes de contextos sin características de vulnerabilidad presentan mayormente niveles medios y bajos. La prueba de chi cuadrado indicó que estas diferencias son significativas $\left(\chi^{2}=8.44 p<0.0015\right.$ Tau $\left.c=-0.370\right)$

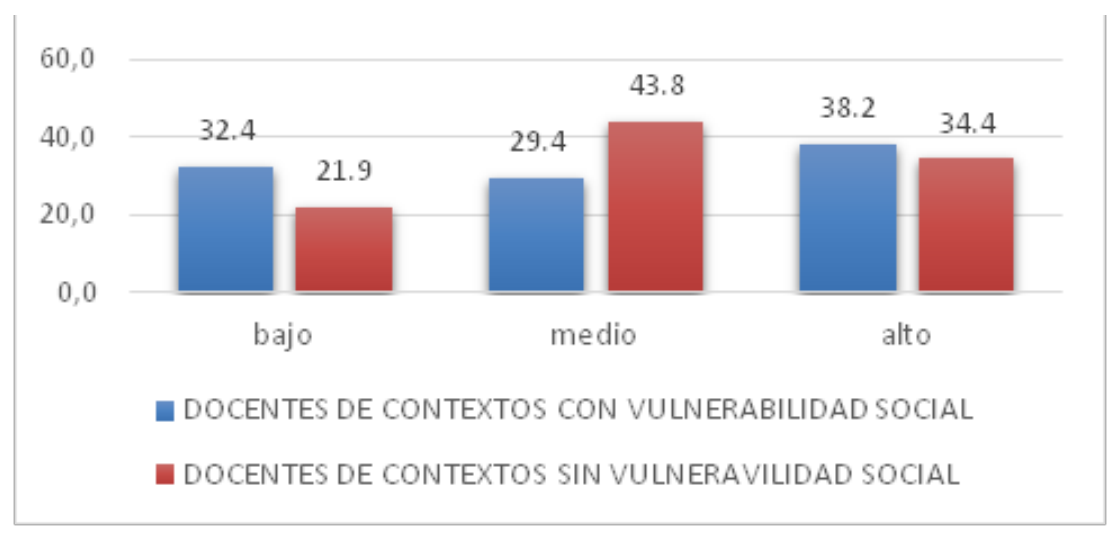

Gráfico 3. Resultados factor 3, "Autoestima y Aprendizaje" según el contexto.

De acuerdo con el Gráfico 3, el grupo de docentes de contextos con vulnerabilidad social presentan mayor prevalencia del rango alto en el factor autoestima y aprendizaje (38.2\%), seguido en segundo lugar por el rango bajo (32.4\%). Por otro lado, el grupo de docentes de contextos sin vulnerabilidad cuenta con valores considerables en el rango medio (43.8\%), seguido del alto (38.2 \%). Es importante señalar que aunque hay diferencias en los rangos, de acuerdo con la prueba de chi cuadrado, estas diferencias no son significativas $\left(\chi^{2}=1.66 p=0.435\right.$ Tau $\left.c=-0.39\right)$

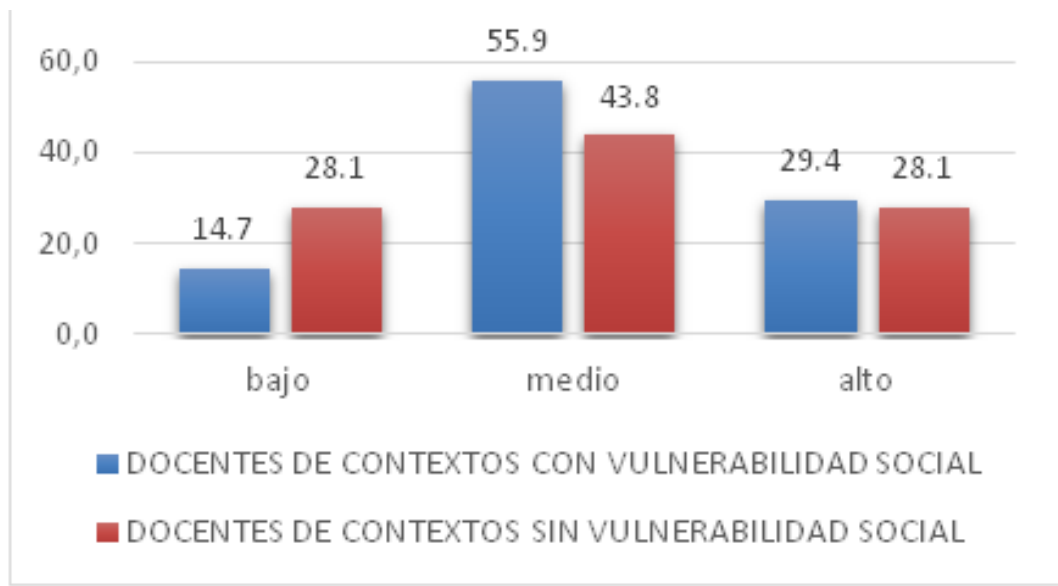

Gráfico 4. Resultados factor 4, "Percepción de apoyo" según el contexto 
Según el Gráfico 4, el factor "Percepción de apoyo", el grupo de docentes de contextos socialmente vulnerables presenta predominantemente valores medios (55.9\%), seguidos por el rango alto (29.4\%). Los docentes de contextos sin vulnerabilidad social también presentan puntajes predominantemente medios (43.8\%), aunque inferiores al grupo de docentes del anterior grupo. Sin embargo, la prueba de chi cuadrado señaló que estas diferencias no son significativas $\left(\chi^{2}=1.89 p<0.38\right.$ Tau $\left.c=-0.106\right)$

Por otra parte, las categorías que emergieron del análisis de la pregunta 4 de la entrevista fueron:

1) No existen diferencias entre contextos: Esta categoría comprende los casos donde el docente manifiesta que no hay diferencias en el trabajo entre contextos con y sin vulnerabilidad social.

2) Existen diferencias en el grupo familiar: Esta categoría aparece cuando el docente comenta que la diferencia principal se encuentra en las características del grupo familiar del alumno.

3) Existen diferencias en el alumno: Se da cuando el docente manifiesta que la diferencia en el trabajo entre un contexto y otro se encuentra en las características del alumno.

4) Existen diferencias en el grupo docente: Aquí el docente encuentra diferencias en diversos aspectos del grupo humano de trabajo.

5) Abordaje de factores emocionales: Según esta categoría, los docentes perciben diferencias en el tipo de problemáticas que debe abordar el docente en relación a aspectos emocionales.

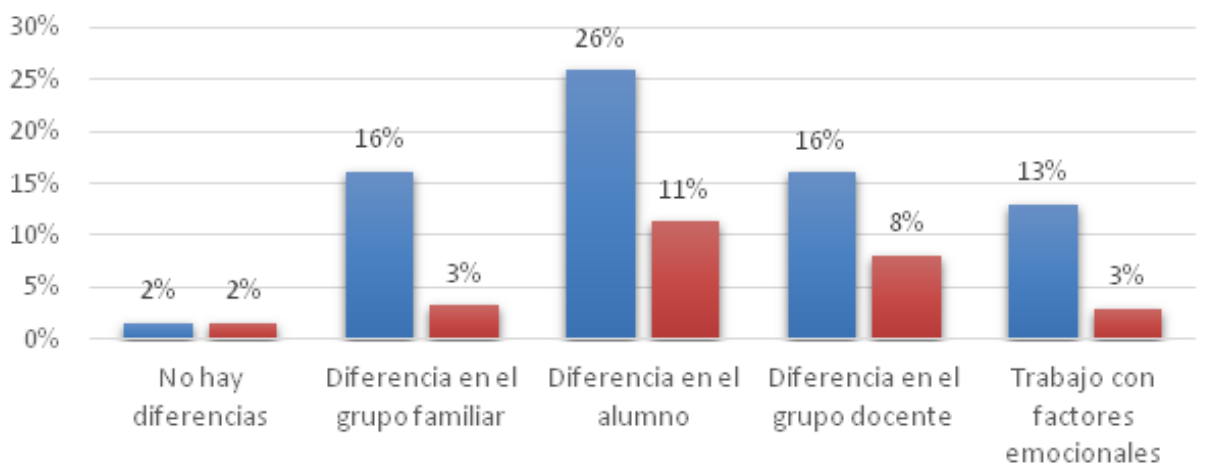

- DOCENTES DE CONTEXTOS CON VULNERABILIDAD SOCIAL

- DOCENTES DE CONTEXTOS SIN VULNERABILIDAD SOCIAL

Gráfico 5. Distribución de las respuestas en relación a las diferencias que puede identificar el docente entre contextos 
De acuerdo con los resultados obtenidos, los docentes de ambos contextos observan que la principal diferencia se encuentra en las características del alumno (Gráfico 5). También opinan que las diferencias se observan en el grupo familiar, como en el grupo docente. Respecto de las características del alumno, ellos consideran que este difiere principalmente en sus características personales, dado que en contextos vulnerables se presenta mayor dificultad en cuanto al acatamiento de las normas de convivencia, menos interés en el aprendizaje cognitivo y una crianza que tiene menor proyección a futuro. Asimismo, los docentes sostienen que en contextos con vulnerabilidad social las familias suelen ser menos participativas en el cumplimiento de lo que se les solicite, suelen ser familias más jóvenes, y en ocasiones, con conflictos intrafamiliares explícitos. Por otra parte, respecto a las diferencias en el grupo docente, muchos manifestaron que en contextos con vulnerabilidad social suele haber mayor cohesión, ya que aun cuando se trabaja con menos recursos materiales, se requiere de un mayor compromiso y vocación del docente.

Finalmente, una cuarta característica la constituyó el trabajo con aspectos emocionales. De este modo, se evidenció que los docentes de contextos socialmente vulnerables estiman que, además de cumplir con los objetivos académicos, deben trabajar sobre factores emocionales de los alumnos, los cuales se relacionan con su falta de apoyo, conflictos familiares, carencias afectivas y crianzas disfuncionales.

Tabla 3.

Prueba T de diferencia entre medias para muestras independientes. Comparación de los 4 factores de resiliencia de los docentes en relación al contexto social.

\begin{tabular}{cccccccc}
\hline FACTOR DE RESILIENCIA & CONTEXTO SOCIAL & N & M & DE & T & P & D \\
\hline \multirow{2}{*}{ Participación significativa } & Con vulnerabilidad & 34 & 161.03 & 12.474 & 1.715 & 0.091 & 0.42 \\
& Sin vulnerabilidad & 32 & 155.72 & 12.673 & & & 0.009 \\
Conducta Prosocial & Con vulnerabilidad & 34 & 77.00 & 7.592 & 2.687 & 0.66 \\
& Sin vulnerabilidad & 32 & 72.31 & 6.498 & & & 0.931 \\
Autoestima y aprendizaje & Con vulnerabilidad & 34 & 21.65 & 1.905 & -0.086 & 0.021 \\
& Sin vulnerabilidad & 32 & 21.69 & 1.891 & & & 0.071 \\
Percepción de apoyo & Con vulnerabilidad & 34 & 31.03 & 3.950 & 1.839 & 0.451 \\
\hline
\end{tabular}

Nota: $n=66 p<0.05$

En la Tabla 3 puede observarse el análisis comparativo de los 4 factores de resiliencia de los docentes en relación al contexto social y según la prueba $t$ de diferencia entre medias. La tabla pone de manifiesto que hubo diferencias significativas en el factor 2 (conducta prosocial), con un tamaño del consi- 
derado adecuado a favor del grupo de docentes de contextos socialmente vulnerables. Además, se observa una tendencia a la significación en el factor 4 (percepción de apoyo), con un tamaño del efecto moderado; igual ocurre en el factor 1 (participación significativa), aunque menos significativa que en el factor 4 (Aron y Aron, 2001; Bordingnon, Galvis, Bertoletti y Trentini, 2017; Navarro Llobel y Pérez, 2000). En el factor 3 (Autoestima y Aprendizaje) no se encontraron diferencias significativas entre los grupos comparados.

\section{DISCUSIÓN}

Siguiendo el objetivo central de la presente investigación, los resultados porcentuales indicaron que el grupo de docentes que trabaja en contextos con vulnerabilidad posee una mayor prevalencia de los niveles medio y alto en los factores "participación significativa" y "conducta prosocial". Por otra parte, el grupo de docentes de contextos sin vulnerabilidad social presentó un nivel predominantemente medio y bajo en los mismos factores. Es útil destacar que la participación significativa propicia las oportunidades de actuación en la toma de decisiones y resolución de problemas, generando sentido de pertenencia. A su vez, la conducta prosocial lleva a comportamientos y respuestas positivas hacia compañeros y alumnos (Guerra, 2010). Puede pensarse que quienes trabajan a diario en entornos vulnerables deben poner en marcha, de un modo más predominante y creativo, recursos tales como participar en las decisiones y ayudarse mutuamente, mientras que en la labor en otros contextos demanda menos la necesidad de organizarse y tomar decisiones vinculadas al riesgo de sus alumnos. Como afirman Henderson y Milstein (2003), estos aspectos constituyen factores de resiliencia que pueden asociarse a indicadores personales e institucionales, que predisponen hacia una adaptación positiva (Luthar, Cicchetti y Becker, 2000; Wolin y Wolin, 1993). Además, la resiliencia no solo reside en los individuos sino en la capacidad de conexión y en las relaciones de apoyo, instancia donde el maestro tiene un papel clave (Day \& Gu, 2014; Gu \& Day, 2007).

En la línea de lo planteado, los resultados obtenidos en la prueba de diferencia entre medias indicaron diferencias significativas a favor del grupo de docentes de contextos socialmente vulnerables en el factor "conducta prosocial", así como una tendencia a la significación en el factor "percepción de apoyo" y en "participación significativa". De este modo, la participación en la toma de decisiones y resolución de conflictos, así como el respaldo institucional y comunitario, son factores claves de resiliencia para afrontar la adversidad comunitaria (Guerra, 2010; 2013; Pérez, García, Gil y Caballer, 2010; Aguaded Gómez y Almeida Pires Cavaco, 2016). Además, a menudo 
la falta de insumos, las carencias edilicias, el déficit en el apoyo desde las entidades gubernamentales, la realidad diaria de conflictos y las características propias de los alumnos y grupos familiares, hacen de la labor un desafío diario. En consecuencia, el apoyo mutuo, el trabajo en equipo y el fomento del encuentro y el consenso se tornan fundamentales. En este sentido, Villalobos y Castelán (2016) afirman que las escuelas son exitosas cuando los educadores potencian la participación y resiliencia en sí mismos, en el aula, en la institución y entre los estudiantes. Conjuntamente, la clave fundamental es que la escuela sea capaz de ofrecer los reforzadores de los factores protectores en la vida de los estudiantes y de los docentes.

Por otra parte, en el segundo grupo de docentes, se observó que las oportunidades de participación y el intercambio de ayuda son moderados o menos frecuentes. A modo de hipótesis, se puede decir que esto se debe a que en este caso los maestros se desenvuelven laboralmente en un contexto que cuyas problemáticas a afrontar no son de la misma índole que las del contexto de riesgo o, dicho de otro modo, las dimensiones que conforman la vulnerabilidad no tienen el mismo peso.

Respecto del factor autoestima y aprendizaje, los resultados no fueron significativos en ninguno de los análisis. Además, la confiabilidad de este factor no fue suficientemente adecuada. Por ende, es importante realizar una revisión de estos ítems y ampliar la muestra en posteriores investigaciones.

Finalmente, el análisis de contenido de la entrevista, que compara las respuestas de los docentes según el contexto, pone el foco principal en la diferencia de las características del alumno y, en segundo lugar, en las familias. De este modo, para los docentes, los niños de contextos socialmente vulnerables poseen más dificultades a la hora de responder a las actividades escolares (en ocasiones por carencias en necesidades básicas tales como la alimentación) y de adaptarse a las normas del grupo. Guerra (2013) plantea que en la actualidad miles de niños y adolescentes se hallan en situación de abandono material y psicológico, de forma tal que la familia no siempre desempeña el rol socializador totalizante que le correspondió en otras épocas (González Gallegos, 2007). Ante esta situación, los maestros deben asumir gran cantidad de funciones que antes le correspondían a la familia y suplir también carencias afectivas. Esto se vincula a la categoría que manifiesta la necesidad de abordar no solo aspectos del aprendizaje cognitivo sino también las dificultades emocionales. Así, el capital humano del grupo docente del establecimiento educativo puede funcionar como un 
factor protector para el tipo de alumno que presenta mayor complejidad. La posibilidad de los docentes de reconocer la característica positiva del grupo en la adversidad evidencia que pueden considerar simultáneamente las adversidades y las potencialidades del conjunto, a partir de una mirada que contribuya al bienestar (Seligman, 2003).

\section{CONCLUSIONES}

Este trabajo tuvo por finalidad conocer factores de resiliencia en un grupo de docentes de contextos de riesgo y en docentes sin esta característica. Los resultados generales indican que el grupo de docentes de contextos socialmente vulnerables cuenta con un potencial levemente mayor que el otro grupo para desarrollar procesos de resiliencia, presentando mejores puntajes en áreas relacionadas con la capacidad para entablar relaciones e interacciones empáticas y de colaboración, el apoyo que perciben por parte de la institución o la comunidad, y la posibilidad de participar en la solución de problemas y toma de decisiones. Estos aspectos son protectores de salud mental.

Por lo tanto, se puede concluir que si bien las características de adversidad del contexto escolar pueden generar muchas dificultades en el quehacer, al mismo tiempo pueden ser potenciadoras de procesos de resiliencia, ya que propician el desarrollo de una serie de factores protectores que en interacción con la adversidad permiten amortiguar el impacto los factores de riesgo. Esto depende de condiciones multidimensionales del propio entorno, donde se interrelacionan aspectos sociales y políticos pero también un conjunto de particularidades de los docentes que tienen que ver con el compromiso, la participación y la sensación de apoyo. Tales elementos constituyen la base para la construcción de redes de protección que pueden constituir los actores sociales que forman parte de las instituciones educativas.

Consideramos, entonces, que los aportes de nuestro trabajo se orientan a promover el desarrollo de potencialidades en las comunidades. De este modo, nuestras intervenciones como profesionales de la salud mental deben tender hacia el fortalecimiento de factores positivos, tales como fomentar la unión y el trabajo en equipo, el apoyo mutuo, activar y crear redes que permitan valorar la labor del docente, el cual puede convertirse en un promotor de la resiliencia grupal.

Es importante marcar, por último, como una limitación de este trabajo, el hecho de haber trabajado con una muestra pequeña y no probabilística, lo 
cual lleva a que los análisis sean un tanto acotados. Por ello, en posteriores estudios, sería interesante ampliar el número de docentes que trabajan en contextos vulnerables en diferentes zonas de la provincia, así como también la muestra de docentes que trabajan en contextos no vulnerables, lo cual permitirá profundizar este tipo de trabajos, orientados a diseñar modos de fortalecimiento de recursos.

Nota de Autores:

Este trabajo se realizó en el marco de un proyecto de investigación denominado "Resiliencia y vulnerabilidad socio-familiar. Un abordaje de la parentalidad y de los recursos socioafectivos y cognitivos en el ámbito escolar". El mismo fue subsidiado por el Consejo de Investigaciones de la Universidad del Aconcagua, Mendoza, Argentina.

\section{REFERENCIAS}

Aguaded Gómez, M. C. \& Almeida Pires Cavaco, N. A. (2016). La resiliencia del docente como factor crucial para superar las adversidades en una sociedad de cambio. Tendencias pedagógicas, 28, 167-180. http://dx.doi.org/10.15366/ tp2016.28.012

Aron, A. \& Aron E. (2001). Estadística para Psicología. 2o Edición. Buenos Aires, Argentina: Prentice Hall. Disponible en https://www.casadellibro.com/libro-estadistica-para-psicologia-2-ed/9789879460665/867761

Bravo, A., H. \& López, P. J. (2015). Resiliencia familiar: una revisión sobre artículos publicados en español. Revista IIPSI, 18(2), 151-170. http://dx.doi.org/10.15381/ rinvp.v18i2.12089

Bordignon, S., Galvis-Aparicio, M. J., Bertoletti, J. \& Trentini, C. M. (2017). Bienestar subjetivo, imagen corporal y funcionalidad de candidatos a cirugía bariátrica o sometidos a la misma. Clínica y Salud, 28, 71-80. https://doi.org/10.1016/j. clysa.2017.02.002

Canto, D., Morelato, G. \& Greco, C. (2013). Resiliencia en el ámbito escolar: estudio preliminar de la percepción de los aspectos familiares y escolares en niños de contextos socialmente vulnerables. Pensando Psicología, 9(16), 33-42. http:// dx.doi.org/10.16925/pe.v9i16.615

Colombo, J. A. \& Lipina, S. (2005). Hacia un programa público de estimulación cognitiva infantil. Fundamentos, métodos y resultados de una experiencia de intervención preescolar. Buenos Aires, Argentina: Paidós.

Colombo, J.A. (2007). Pobreza y Desarrollo Infantil. Buenos Aires, Argentina: Paidós.

Cyrulnik, B. (2009). La resiliencia o la capacidad humana de superar pérdidas, crisis, adversidades o traumas. Madrid, España: Institud Français.

D. G. E. (2017). Dirección General de Escuelas (D.G.E.). Sistema de Información Gestión Administrativa de Escuelas. Recuperado de: http://bases.mendoza.edu. ar/intranet/portal_con_esc.asp

Day, C. \& Gu, Q. (2014). Resilient teachers, resilient schools. London: Routledge.

Day. C. \& Hong (2016). Influences on the capacities for emotional resilience of teachers in schools serving disadvantaged urban communities: Challenges of living on the edge. Teaching and Teacher education, 59, 115-125. https://doi. org/10.1016/j.tate.2016.05.015 
Escarbajal-Frutos, A., Izquierdo-Rus, T. \& López-Martínez, O. (2014). Análisis del bienestar Psicológico en grupos en riesgo de exclusión social. Anales de Psicología, 30(2), 541-548. http://dx.doi.org/10.6018/analesps.30.2.156591

Golovanevsky, L. (2007a). Vulnerabilidad Social: una Propuesta para su Medición en Argentina. Revista de Economía y Estadística, 45(2), 53-94. Disponible en https:// revistas.unc.edu.ar/index.php/REyE/article/view/3840/6780

Golovanevsky, L. (2007b). Vulnerabilidad y transmisión intergeneracional de la pobreza. Un abordaje cuantitativo para Argentina en el siglo XXI (Tesis de Doctorado). Universidad de Buenos Aires, Buenos Aires, Argentina. Disponible en http://bibliotecadigital.econ.uba.ar/?c=tesis\&a=d\&d=1501-1179_GolovanevskyL

Gómez, E. \& Kotliarenco, M. (2010). Resiliencia Familiar: un enfoque de investigación e intervención con familias multiproblemáticas. Revista de Psicología, 19(2), 103-132. https://doi.org/10.5354/0719-0581.2010.17112

González Gallegos, V. (2007). La educabilidad y la resiliencia en un grupo étnico. XXVI Congreso de la Asociación Latinoamericana de Sociología, Guadalajara. Disponible en http://cdsa.aacademica.org/000-066/986

Greco, C., Morelato, G. \& Ison, M. (2007). Emociones positivas: una herramienta psicológica para promocionar el proceso de resiliencia infantil. Psicodebate, (7), 1-14. http://dx.doi.org/10.18682/pd.v7i0.429

Gu, Q. \& Day, C. (2007). Teachers' resilience: A necessary condition for effectiveness. Teaching and Teachers Education, 23(8), 1302 - 1316. https://doi.org/10.1016/j. tate.2006.06.006

Guerra, J. (2010). Niveles de Resiliencia en docentes de Inicial, Primaria y Secundaria de la Región Callao (Tesis de maestría). Universidad San Ignacio de Loyola, Perú. Disponible en http://alicia.concytec.gob.pe/vufind/Record/USIL_ b9bd976c6286f3aec7ede3c1dc55f73b

Guerra, J. (2013). Construcción, validez y confiabilidad de la Escala de Resiliencia Docente. Propósitos y Representaciones. Revista de Psicología y Educación de la USIL, 1(1), 59-86. http://dx.doi.org/10.20511/pyr2013.v1n1.7

Henderson, N. \& Milstein, M. (2003). Resiliencia en la escuela, 2o edición. Buenos Aires, Argentina: Paidós.

Hernández Sampieri, R., Fernández Collado, C. \& Baptista Lucio, P. (2010). Metodología de la Investigación. 5o edición. Mexico: Mc Graw Hill.

Herscovici Raush, C. (2011). Resiliencia familiar y el énfasis en los recursos del sistema. Cultura, Educación y Sociedad, 2(1), 105-110. Disponible en http://revistascientificas.cuc.edu.co/index.php/culturaeducacionysociedad/article/view/938

Ison, M. S. (2004). Características familiares y habilidades sociocognitiva en niños con conductas disruptivas. Revista Latinoamericana de Psicología, 36(2), 257-268. Disponible en https://dialnet.unirioja.es/servlet/articulo?codigo=1199062

Ison, M., Morelato, G., Carrada, M., Espósito, A., Greco, C., Maddio, S. \& Korzeniowski, C. (2007). Programa de Intervención para estimular atención sostenida y habilidades cognitivas en niños con disfunción atencional. En C. Richaud y M. Ison (Comp.), Avances en investigación en Ciencias del Comportamiento en Argentina. Mendoza: Universidad del Aconcagua. Disponible en http://www.redalyc.org/ pdf/2990/299023516009.pdf

Korzeniowski, C. (2011). Desarrollo evolutivo del funcionamiento ejecutivo y su relación con el aprendizaje escolar. Revista de Psicología, 7(13), 7-26. Disponible en http://bibliotecadigital.uca.edu.ar/greenstone/cgi-bin/library.cgi?a=d\&c=Revistas\&d=desarrollo-evolutivo-funcionamiento-ejecutivo 
Lacunza, A. B., Castro-Solano, A. \& Contini, N. (2009). Habilidades sociales preescolares: una escala para niños de contextos de pobreza. Revista de Psicología, 27(1), 3-28. Disponible en http://revistas.pucp.edu.pe/index.php/psicologia/article/ view/239

López Hidalgo, I. (2012). Niveles de resiliencia en los docentes de primaria de las instituciones públicas de Ventanilla y Callao (Tesis de Maestría). Universidad San Ignacio de Loyola, Lima, Perú. Disponible en http://alicia.concytec.gob.pe/ vufind/Record/USIL_06556b699c65528b94f5ce750984e835

Luthar, S., Cicchetti, D. \& Becker, B. (2000). The construct of resilience: A critical evaluation and guidelines for future work. Child Development, 71, 543-562. https://doi.org/10.1111/1467-8624.00164

Mariñelarena-Dondena, L. (2016). Recepción de la Psicología Positiva en la Argentina: un estudio socio-bibliométrico de artículos de revistas científicas. Revista Argentina de ciencias del comportamiento, 8(1), 27-42. Disponible en https:// revistas.unc.edu.ar/index.php/racc/article/view/12666/Dondena4

Marzonetto, G. L. (2015). Jugando con desventaja. La estratificación de los servicios de Cuidado y desarrollo infantil en la Ciudad Autónoma de Buenos Aires (Tesis de Maestría). Georgetown University, Universidad en Washington D. C., Estados Unidos. Disponible en https://repository.library.georgetown.edu/ handle/10822/1040701

McClelland, M., Cameron, C., Connor, C., McDonald, F., Carrie, L., Jewkes, A. \& Morrison, F. (2007). Links between behavioural regulation and preschoolers' literacy, vocabulary, and math skills. Developmental Psychology, 43(4), 947-959. http://dx.doi.org/10.1037/0012-1649.43.4.947

Morelato, G. (2011a). Maltrato infantil y desarrollo: hacia una revisión de los factores de resiliencia. Pensamiento Psicológico, 9(17), 83-96. Disponible en http://www. scielo.org.co/scielo.php?script=sci_arttext\&pid=S1657-89612011000200008

Morelato, G. (2011b). La Resiliencia en el Maltrato Infantil: aportes para la comprensión del proceso desde un modelo ecológico. Revista de Psicología, 29(2), 203-224. Disponible en http://revistas.pucp.edu.pe/index.php/psicologia/ article/view/2467

Morelato, G. (2014). Resiliencia y maltrato infantil desde un enfoque ecológico: importancia del contexto para el desarrollo. En: M.B. Marsonet, J.M. Vitaliti y M. Quintero (Comp.), Miradas y Experiencias Interdisciplinarias. Produciendo nuevas vinculaciones y conocimientos sobre el Maltrato a la Niñez y Adolescencia desde un ámbito público de la Provincia de Mendoza. Bs. As: Cauquén. Disponible en http://www.scielo.org.pe/pdf/psico/v29n2/a01v29n2.pdf

Munist, M., Santos, H. \& Kotliarenco, M. (1998). Manual de identificación y promoción de la resiliencia en niños y adolescentes. OPS. OMS: Fundación Kellog. Disponible en https://www.psyciencia.com/manual-resiliencia-ninos-adolescentes/

Navarro, M. D. F., Llobel, J. P. \& Pérez, J. F. G. (2000). Tamaño del efecto del tratamiento y significación estadística. Psicothema, 12(Suplemento), 236-240. Disponible en http://www.psicothema.es/pdf/555.pdf

Pérez, R. M., García, M., Gil, J. \& Caballer, A. (2010). ¿Qué es la resiliencia? Hacia un modelo integrador. Fòrum de Recerca, 15, 231-248. Disponible en http:// repositori.uji.es/xmlui/handle/10234/77669

Pinto Cortez, C. (2014). Resiliencia Psicológica: una aproximación hacia su conceptualización, enfoques teóricos y relación con el abuso sexual infantil. Summa Psicológica UST, 2(11), 19-33. Disponible en http://vufind.uniovi.es/Record/ ir-ART0000733966 
Ríos, V. \& Andes, K. (2016). Resiliencia y salud adolescente: una apreciación de los riesgos y fortalezas en el Bañado Sur de Asunción, Paraguay. Revista Salud Pública Paraguay, 1(6), 16-23. Disponible en http://www.ins.gov.py/revistas/index.php/ rspp/article/view/344/268

Rosemberg, C. R. \& Stein, A. (2009). Vocabulario y alfabetización temprana. Un estudio del entorno lingüístico en hogares de poblaciones urbano-marginadas. En M.C. Richard y E. Moreno (Ed). Investigación en Ciencias del Comportamiento. Avances Iberoamericanos. Buenos Aires: CIIPME-CONICET. Disponible en http:// www.academia.edu/3684090/Vocabulario_y_alfabetizaci\%C3\%B3n_temprana. Un_estudio_del_entorno_ling\%C3\%BC\%C3\%ADstico_en_hogares_de_poblaciones_urbano_marginadas

Segretin, M. S., Lipina, S. J. \& Colombo, J. A. (2007). Intervenciones para niños en riesgo social: análisis de predicción de mejora cognitiva. En M. C. Richaud \& M. $\mathrm{S}$. Ison (Comp.), Avances en investigación en ciencias del comportamiento en Argentina. Argentina: Universidad del Aconcagua. Disponible en https://www. researchgate.net/publication/236869356_Intervenciones_para_ninos_en_ riesgo_social_Analisis_de_prediccion_de_mejora_cognitiva

Seligman, M.E.P. (2003). La auténtica felicidad. España: Vergara. Disponible en https://books.google.com.co/books/about/La_aut\%C3\%A9ntica_felicidad. html?id=wA4JDgAAQBAJ\&source=kp_cover\&redir_esc $=y$

Siquier de Ocampo, M., García Arzeno, M., Grassano, E. \& colaboradores. (1997). Las técnicas proyectivas y el proceso psicodiagnóstico. Argentina: Nueva visión. Disponible en https://www.amazon.es/Tecnicas-Proyectivas-El-Proceso-Psicodiagnostico/dp/9506020027

Valdés Arriagada, M. \& Spencer Contreras, R. (2011). Influencia del nivel socioeconómico familiar sobre el desarrollo psicomotor de niños y niñas de 4 a 5 años de edad de la ciudad de Talca-Chile. Theroria, 20(2), 29-43. Disponible en http:// www.ubiobio.cl/miweb/webfile/media/194/v/v20-2/4.pdf

Villalobos Torres, E. \& Castelán García, E. (2006). Resiliencia: el arte de navegar en los torrentes. Revista panamericana de pedagogía, 8, 287-303. Disponible en https://dialnet.unirioja.es/servlet/articulo?codigo $=2796805$

Wolin, S. J. \& Wolin, S. (1993). The resilient Self: How Survivors of Troubled Families Rise above Adversity. Nueva York: Villard. Disponible en https://books.google. com.co/books/about/The_Resilient_Self.html?id=7UF3-Wjg4SoC\&source=kp_ cover\&redir_esc=y autor o el licenciante.

\section{(cc) BY}

